\title{
Clinical Study on the Treatment of Metastatic Malignant Bowel Obstruction with Transgastric Intestinal Obstruction Catheter Arrangement Small Intestinal Enterostomy
}

\author{
Song Bin ${ }^{1}$, Du Juan ${ }^{2}$, Hu Haixia ${ }^{1}$, and Su Yanzhuo ${ }^{1, *}$ \\ ${ }^{1}$ Department of Gastrointestinal, Colorectal and Anal Surgery, China-Japan Union Hospital of Jilin \\ University, Changchun, Jilin 130033, China; \\ ${ }^{2}$ Internal Medicine 2, The Tumor Hospital of Jilin Changchun, Jilin 130012, P.R. China \\ *corresponding author
}

Keywords: intestinal obstruction catheter; bowel enterostomy; malignant bowel obstruction

\begin{abstract}
To explore the value of application of transgastric intestinal obstruction catheter arrangement small intestinal enterostomy in the treatment of metastatic malignant bowel obstruction, the paper selected 15 cases of patients with metastatic malignant bowel obstruction who meet the inclusion criteria, they were performed with a transgastric poke with bowel obstruction catheter for an arrangement of small intestine and intestinal enterostomy; the effect of perioperative period was observed, and a post-operation follow-up was conducted to understand the relief of obstruction and survival time. Results show all 15 patients completed operation, and the operation was successfully done, the operation time was $85-132 \mathrm{~min}$ (averagely $118.7 \mathrm{~min}$ ); the recovery time of gastrointestinal function was $2-7 \mathrm{~d}$, median time was $3.5 \mathrm{~d}$; hospitalization time was 8-33d, median hospitalization time was $17.8 \mathrm{~d}$; there was one case of death in the peri-operative period. 14 cases were followed up, the follow-up time was 3 - 38 months (averagely 20.7 months), survival time was 3 -38 months, median survival time was 15.4 months, the benefit remission rate was 93.3\% (14/15). In a word, a reasonable selection and application of transgastric bowel obstruction catheter arrangement small intestinal enterostomy can relieve the bowel obstruction in part of the patients, and oral feeding can delay the time of reobstruction, extending the survival time.
\end{abstract}

\section{Introduction}

Malignant bowel obstruction refers to the intestinal obstruction caused by primary or metastatic malignant tumor, and it is a common complication and cause of death in patients with advanced tumor [1]. The intestinal obstruction caused by metastatic malignant tumor is often caused by abdominal multiple metastasis, which is easy to relapse after operation, and a conservative treatment has difficulty in relieving the obstruction, which is currently a difficult problem in the treatment of advanced cancer. Based on the role of bowel obstruction catheter in the conservative treatment and arranged surgical treatment of complex intestinal obstruction [2-3], from June 2007 to August 2014, a transgastric bowel obstruction catheter arrangement small intestinal enterostomy was used to treat metastatic malignant intestinal obstruction in our hospital, and a certain clinical result were achieved, which is now reported as follows:

\section{Clinical data}

\subsection{Case inclusion criteria}

Patients with metastatic malignant bowel obstruction were mostly in their advanced stage and had a limited survival time, with a view to the benefits of patients and reduction of complications, the following patients were included into the group: (1) devoid of dyscrasia and severe malnutrition, (2), devoid of heart, lung and kidney failure; (3) without a history of surgery in the last 3 months; (4) the patient does not reject bowel enterostomy, and has a strong desire for oral feeding; (5) liver, lung, 
brain and other main functional organs are free of metastasis ; (6) the possibility of digestive tract obstruction caused by single tumor is excluded, (7) no effect of relief is achieved by trans-nasal indwelling bowel obstruction catheter and other conservative treatments, (8) without severe anemia, peritonitis, fever and serious infection.

\subsection{General data}

All 15 cases were diagnosed before operation as conforming to the inclusion criteria. Among them there were 7 cases of male and 8 cases of female; with an age of onset ranging from 45 to 67, median age was 56.7 years old. The interval between the development of symptom of bowel obstruction and the operation was 4 days to 1 month. 9 Cases ( 4 cases of patient had been performed with ovarian cancer radical resection, 3 cases had been performed with radical resection of gastric cancer, 2 cases had been performed with radical resection of colorectal cancer) had been performed with laparotomy to conduct a resection of primary tumor and a post-operative chemotherapy, 1 case of patient was in a state of post-operation of local resection of skin cancer , 5 cases were found to be primary attack case, (3 case of extensive metastasis of ovarian cancer in abdominal cavity, 2 case of extensive metastasis of colon cancer in abdominal cavity). Before the operation, 5 cases were found to have a moderate anemia, and 4 cases were found to have a mild anemia. Preoperative albumin : $28 \sim 36 \mathrm{~g} / \mathrm{L}$. Routine examinations like abdominal ultrasonography, whole-abdomen CT, gastroscopy and colonoscopy were performed, the female patients underwent a gynecological ultrasound examination, and a small intestine radiography was performed through trans-nasal indwelling bowel obstruction catheter. The intestinal obstruction catheter is made of three cavities (front airbag, Posterior airbag) and the intestinal obstruction catheter being used was the 3-cavity-2-bag (front air bag, back air bag) bowel obstruction catheter that is produced by Japanese CREATEMEDIC company.

\subsection{Method}

\subsubsection{Preoperative basic treatment}

The patients were administered with fluid infusion, correction of anemia, correction of hypoproteinemia, nutritional support, enema, correcting acid-base imbalance and ion disorder before the operation, all cases before operation were corrected to the point where their hemoglobin was more than 90g/l and albumin more than 35g/l. After admission they were immediately administered with a small intestine decompression after the bowel obstruction catheter was indwelled, the bowel obstruction catheter passed through smoothly in 10 cases, to the depth of 2.3 $\sim 2.9 \mathrm{~m}$; and the intestinal obstruction catheter reached to the depth of $60 \mathrm{~cm}$ and $65 \mathrm{~cm}$ in two cases respectively. In 15 cases of patient, the abdominal distension and abdominal pain were relieved after decompression, and the duration of conservative treatment was $5 \sim 10 \mathrm{~d}$, a meglumine diatrizoate radiography was carried out through the bowel obstruction catheter and it was found that, the distal end was still obstructed. On the day of operation, the intestinal obstruction catheter was withdrawn, and disinfected by povidone iodine for standby use.

\subsubsection{Intraoperative operation}

All cases were performed with a general anesthesia, all of them were poked through the greater curvature of stomach in order to arrange the intestinal obstruction catheter to the position of small intestinal enterostomy, it is required that, more than $2 \mathrm{~m}$ of small intestine that is free of obvious stenosis and able to allow the $10 \mathrm{~mL}$ water bag of bowel obstruction catheter to pass through be retained. (1) treatment in the case of absence of pylorus and duodenal stenosis. A lateral incision with a diameter of about $0.5 \mathrm{~cm}$ was performed through the greater curvature of stomach, and the head of intestinal obstruction catheter was inserted into the gastric cavity through the abdominal wall. The intestinal obstruction catheter was introduced into the small intestine through the pylorus and duodenum till it reached the fully obstructed part, and the intestinal juice was sucked by negative pressure suction at the same time. If the length of intestine at the proximal end of obstruction is more than $2 \mathrm{~m}$, then the normal small intestine $10 \mathrm{~cm}$ above the obstruction is raised 
through the lateral abdominal wall out of the stoma, and at the same time the intestinal obstruction catheter was retained in intestine cavity.

If the length of intestine at the proximal end of obstruction is less than $2 \mathrm{~m}$, the intestinal canal at the fully obstructed position should be resected and an anastomosis should follow, or the obstructed position should be put aside to perform a short-circuit operation, followed by a small intestine enterostomy. Finally, the gastric incision should be embedded with double-pouch method and fixed in the abdominal cavity and at the abdominal wall skin. (2) the treatment in the case of presence of pylorus and duodenal stenosis. A gastrointestinal anastomosis should be firstly performed, then the bowel obstruction catheter should be introduced into the small intestine through gastrointestinal anastomotic stoma till it reach the fully obstructed part, the remaining treatment is the same as the treatment in the case of absence of pyloric and duodenal stenosis.

\subsubsection{Result judgment criteria}

The criteria of benefit and remission should be subject to the patient being capable of normal oral feeding, free of relapse of bowel obstruction and surviving for more than 60 days.

\subsection{Result}

\subsubsection{Intraoperative condition}

Operation duration: 85-132min (averagely $118.7 \mathrm{~min}$ ). 4 cases of patient were performed with small intestine enterostomy alone, 4 cases of patient were performed with resection of small intestine and anastomotic small intestine enterostomy; 3 cases were performed with small intestine putting-aside and side-to-side anastomotic small intestine enterostomy; 4 cases of patient with ovarian cancer complicated with duodenal stenosis and jejunum proximal stenosis were performed with gastrointestinal anastomosis small intestine enterostomy.

\subsubsection{Postoperative recovery}

The recovery time of gastrointestinal function for this group of patients was 2-7d, median time was 3.5d; hospitalization time was 8 -33d, median hospitalization time was $17.8 \mathrm{~d}$.

\subsubsection{Death and complication}

One case of patient died from a postoperative small intestine fistula and disruption of wound 21 days after operation; 5 cases were found to have incision infection; 1 case was found to have infection at the position of stoma, and exudation of gastric juice was found, which was relieved after repeated dressing change; 1 case was found to have intra-abdominal abscess, which was relieved by drainage and anti-infection.

\subsubsection{Postoperative follow-up result}

The patients were followed up by outpatient service and telephone follow-up on month 1, 2, 3, 6 , 12, 24 and 36 after operation respectively, with an exception that 1 case of patient died on day 21 after operation, the remaining 14 cases of patient were all followed up, follow-up duration was 3 38 months (averagely 20.7 months). Survival time was 3 -38 months, median survival time was 15.4 months. 2 death was reported during the follow-up period, as a result of re-obstruction. In this group, 14 cases of patient were benefited and remitted and the benefit remission rate was $93.3 \%$.

\section{Discussion}

Malignant intestinal obstruction is a common complication in the late stage of malignant tumor, including the intestinal obstruction caused by primary or metastatic malignant tumor. Different from the primary malignant intestinal obstruction, the patients with metastatic malignant bowel obstruction usually have multiple lesions, which are often scattered over the whole abdominal cavity, the obstruction mostly occurs at the small intestine, it is common in the terminal stage of ovarian cancer, colorectal cancer, gastric cancer and other tumors, and is currently a difficult problem faced in the treatment of advanced cancer [6]. 
The current treatment methods mainly include traditional surgery, placement of extendable stent, drug therapy, percutaneous endoscopic gastrostomy , nasogastric tube drainage and total parenteral nutrition, the traditional operations usually prefer stage- 1 intestinal anastomosis and bypass surgery, according to the conservative view, the operation is only suitable for mechanical obstruction and/or the obstruction with a limited tumor and single site, and for the patients who may benefit from further chemotherapy and anti-tumor therapy; extendable Stent therapy is usually used in the treatment of the pyloric and proximal small intestine and colonic obstruction, but it is regarded as a stent therapy contraindication for the patients with multiple obstructions and extensive metastasis in abdominal cavity [6]. Long-term intravenous nutrition, which is expensive and at a high risk of infection, blood glucose disorders and other complications, can only ensure the supply of nutrition and can not solve the obstruction problems. The application of Anti-secretion drugs, antemetics, glucocorticoid and other drugs can only mildly alleviate abdominal distension, reduce some of the digestive fluids, and must be used in combination with gastrointestinal decompression and intravenous nutrition.

This study used intestinal obstruction catheter to perform a nasal small intestine decompression before the operation in order to relieve the patient's abdominal distension, and to avoid emergency operation, preoperative adjustment time was $5 \sim 9 \mathrm{~d}$, which has won time for correcting the hypoproteinemia . In this group, the level of preoperative albumin was maintained above $35 \mathrm{~g} / \mathrm{l}$, and at the same time a reasonable supplement of nutrition was administered to correct anemia and electrolyte disorder, helping to improve the overall physiological function disorder caused by obstruction. Some studies have shown that an albumin level that is lower than $35 \mathrm{~g} / \mathrm{l}$ can be used as a poor prognostic factor for patients with malignant intestinal obstruction, suggesting that patients with malignant intestinal obstruction whose albumin level is less than $35 \mathrm{~g} / \mathrm{L}$ should be given a targeted treatment as early as possible, which may improve the overall prognosis of patients.

12 cases did not develop intestinal obstruction again till their death, suggesting that, the retention of bowel obstruction catheter plays a role of stent in the intestinal cavity, to a certain extent, it has slowed the growth of tumor towards the cavity, kept the proximal intestinal tract basically unobstructed and postponed the re-stenosis . Literature has reported that the throat discomfort, pain and other complications brought by the nasal indwelling catheter are $98.1 \%$ likely to happen, some patients have a serious reaction and can not tolerate it. We use trans-gastric enterostomy indwelling catheter to avoid such complications that affect the quality of life of patients, and it can be carried for a long time and doesn't fall off.

In a word, metastatic malignant bowel obstruction is a clinical difficulty, it is necessary to combine the patients ' actual conditions and strictly grasp the indications; rationally choosing the use of transgastric bowel obstruction catheter arrangement small intestinal enterostomy can relieve part of the patients from obstruction and promote oral feeding while extending the time of reobstruction and survival time and improving the survival quality.

\section{References}

[1] Selby D, Wright F, Stilos K, et al. Room for improvement? A quality-of-life assessment in patients with malignant bowel obstruction. Palliat Med, 2010, 24(1): 38-45.

[2] Niu lujie, Sun libo, Zhang Bing et al. Clinical analysis of 38 cases where the bowel obstruction catheter is used to treat low bowel obstruction. Chinese Journal of General Surgery, 2010, 25(1): 79-80.

[3] Jin Yinzhi, Sun Libo, Song Bing. the therapeutic effect of bowel obstruction catheter on adhesive intestinal obstruction. Chinese Journal of Gastrointestinal Surgery, 2009, 12(6): 580.

[4] Dolan EA. Malignant bowel obstruction: a review of current treatment strategies. Am J Hosp Palliat Care, 2011, 28(8): 576-582. 
[5] Yu Shiying, Wang Jiejun, Wang Jinwan et al. expert consensus on the treatment of patients with advanced cancer complicated with bowel obstruction. Chinese Journal of Tumor, 2007, 29(8): 637640.

[6] Zhang Qifan, Zheng Hongqun, Sun Lingyu. Diagnosis and treatment of malignant intestinal obstruction caused by peritoneal metastasis of gastric cancer and nutritional support strategies. Chinese Oncology Clinical, 2014, 41(12): 749-752.

[7] Hisanaga T, Shinjo T, Morita T, et al. Multicenter prospective study on efficacy and safety of octreotide for inoperable malignant bowel obstruction. Jpn J Clin Oncol, 2010, 40(8): 739-745.

[8] Wang Aman, Ning Zheng, Zhou Tao et al. Prognostic analysis of advanced tumors complicated with malignant intestinal obstruction. Modern Oncology medicine, 2014, 22(2): 379-383.

[9] Barbot AC, Mussault P, Ingrand P, et al. Assessing 2-month clinical prognosis in hospitalized patients with advanced solid tumors. J Clin Oncol, 2008, 26(15): 2538-2543.

[10] He Xuehong, Mao Aiwu, Fang Shiming et al. Treatment of benign and malignant intestinal obstruction by DSA-guided nasal inserted intestinal obstruction catheter. Western medicine, 2013, 25(10): 1548-1550. 